Bull. Austral. Math. Soc.

Vol. 74 (2006) [161-175]

\title{
SEMILINEAR ELLIPTIC NEUMANN PROBLEMS WITH RAPID GROWTH IN THE NONLINEARITY
}

\author{
JASON R. LOOKER
}

\begin{abstract}
The existence and regularity of solutions to semilinear elliptic Neumann problems are investigated. Motivated by the Poisson-Boltzmann equation of biophysics and semiconductor modeling, the nonlinearity is assumed to be a continuous, strictly monotone increasing function that passes through the origin with asymptotically superlinear and unbounded growth. Pseudomonotone operator theory is utilised to establish the existence and uniqueness of a weak solution in the Sobolev space $W^{1,2}$. With an additional assumption on the nonlinearity, we show that this weak solution belongs to $W_{\mathrm{loc}}^{2,2} \cap L^{\infty}$.
\end{abstract}

\section{INTRODUCTION}

The existence of a solution to the nonlinear Poisson equation with a Neumann boundary condition is sought in the Sobolev space $W^{1,2}$ (also known as $H^{1}$ ). The nonlinearity is a continuous, strictly monotone increasing function that passes through the origin with asymptotically superlinear and unbounded growth. Higher regularity of the solution will be investigated under the additional requirement that the nonlinearity grows faster than any polynomial.

The nonlinear Poisson equation

$$
-\Delta u+f(u)=g,
$$

has been studied extensively by numerous researches; see $[5,20,22,24]$ for an overview. Existence has been established when the nonlinearity is in some sense bounded, or is unbounded but obeys a bounded growth condition; for example $[9,10,11,14,18,19]$. These conditions can be relaxed at the expense of restricting the solution space. If the nonlinearity has unbounded growth, then there exists a solution of the Dirichlet problem in the Sobolev space $W_{0}^{1,2}$; see $[2,6,7,13,16]$. Strongly nonlinear elliptic boundary

Received 11th October, 2005

The author would like to thank Kris Wysocki for his many helpful suggestions. This work would not have come to fruition without his input. I also thank Steven Carnie for his assistance in the preparation of this article.

The Particulate Fluids Processing Centre is an Australian Research Council Special Research Centre. Copyright Clearance Centre, Inc. Serial-fee code: 0004-9727/06 \$A2.00+0.00. 
value problems where no growth restrictions are imposed on the nonlinearity have also been investigated in $[12,23]$. For instance in [12], a solution is shown to exist in the space $W^{1, q}(1<q<\infty)$ such that $f(u)$ and $f(u) u$ are elements of the Lebesgue space $L^{1}$. Unfortunately, the nonlinear differential operators considered in these papers are assumed to obey a coercivity condition, which does not seem to be applicable to the Laplacian when a solution is sought in $W^{1,2}$, as opposed to $W_{0}^{1,2}$. It appears that noncoercive semilinear elliptic Neumann problems with unbounded growth in the nonlinearity, have not been extensively studied in the literature.

An example of a nonlinear Poisson equation with rapid and unbounded growth in the nonlinearity is the Poisson-Boltzmann equation that arises in biophysics [13]. In particular it is ubiquitous in colloid science $[15,21]$. There is also a close link between this equation and the phenomenological theory of electron and hole transport in semiconductors $[16,21]$. The Poisson-Boltzmann equation with a constant charge boundary condition is given by

$$
\begin{aligned}
-\Delta u(x) & =4 \pi \sum_{j=1}^{N} z_{j} n_{j}^{\infty} \exp \left(-z_{j} u(x)\right) & & \text { in } \Omega \\
\frac{\partial u}{\partial n} & =-\sigma & & \text { on } \partial \Omega,
\end{aligned}
$$

where $u: \Omega \rightarrow \mathbb{R}$ represents the equilibrium electrostatic potential, $z_{j} \in \mathbb{Z} \backslash\{0\}$ are the valencies and $n_{j}^{\infty}>0$ the bulk ionic concentrations of the $j$ th ionic species $(j=1, \ldots, N)$ of the electrolyte, while $\sigma$ is the (constant) surface charge density. The operator $\partial / \partial n$ is the normal derivative on $\partial \Omega$. All quantities have been rendered dimensionless for convenience. The $z_{j}$ and $n_{j}^{\infty}$ satisfy the bulk electroneutrality condition

$$
\sum_{j=1}^{N} z_{j} n_{j}^{\infty}=0,
$$

so that if $\sigma=0$, the unique solution to Equation (1) is $u=0$.

Motivated by the Poisson-Boltzmann system, the following nonlinear Poisson equation will be studied

$$
\begin{aligned}
-\Delta u(x)+f(u(x)) & =g(x) & & \text { in } \Omega, \\
\frac{\partial u}{\partial n} & =h & & \text { on } \partial \Omega,
\end{aligned}
$$

where $g \in H^{1}(\Omega) \cap L^{\infty}(\Omega)$ and $h \in L^{2}(\partial \Omega) \cap L^{\infty}(\partial \Omega)$. Equation (5) is assumed to hold in the trace sense $[4,17]$, while the domain $\Omega \subset \mathbb{R}^{d}$ with $d \geqslant 1$ is bounded and of class $\mathcal{C}^{0,1}$ (for example, piecewise Lipschitz) [17]. The assumptions on the nonlinear function $f$ are:

(A1) $f: \mathbb{R} \rightarrow \mathbb{R}$ with $f$ continuous; 
(A2) $f(0)=0$;

(A3) $f$ is strictly monotone increasing, that is,

$$
(f(s)-f(t))(s-t)>0,
$$

for all $s, t \in \mathbb{R}$ with $s \neq t$;

(A4) there exist $C_{f}^{+}, C_{f}^{-}>0$ such that

$$
\lim _{t \rightarrow+\infty} \frac{f(t)}{t} \geqslant C_{f}^{+} \text {and } \lim _{t \rightarrow-\infty} \frac{f(t)}{t} \leqslant C_{f}^{-}
$$

(A5) for higher regularity we require

$$
\lim _{|t| \rightarrow \infty} \frac{|f(t)|}{|t|^{n}} \rightarrow \infty
$$

for all $n \in \mathbb{N}$.

Observe that $|f(t)|$ grows without bound as $t \rightarrow \pm \infty$.

We wish to prove that the nonlinearity of the Poisson-Boltzmann equation,

$$
f(t ; z)=-4 \pi \sum_{j=1}^{N} z_{j} n_{j}^{\infty} \exp \left(-z_{j} t\right),
$$

obeys assumptions (A1)-(A5); to exhibit this nonlinearity's dependence on the valency $z_{j}$, we have adopted the notation $f(t ; z)$ where $z=\left(z_{1}, z_{2}, \ldots, z_{N}\right)$. Commencing with (A1), since $f$ is smooth, it must also be continuous. Equation (3) gives $f(0 ; z)=0$. Differentiating $f$,

$$
f^{\prime}(t ; z)=4 \pi \sum_{j=1}^{N} z_{j}^{2} n_{j}^{\infty} \exp \left(-z_{j} t\right)>0,
$$

for all $t \in \mathbb{R}$, which follows from $n_{j}^{\infty}>0$. Note that $f$ does not have to be differentiable for (A3) to be true. Assumption (A4) follows from (A5) with $n=1$. To prove (A5), we first observe that

$$
f(t ; z)=-f(-t ;-z) .
$$

Let $t<0$. The monotonicity of $f$ plus $f(0)=0$ give

$$
\begin{aligned}
|f(t ; z)| & =-f(t ; z) \\
& =f(-t ;-z) \\
& =f(|t| ;-z) .
\end{aligned}
$$

Now let $t \geqslant 0$. The monotonicity of $f$ plus $f(0)=0$ also ensure

$$
|f(t ; z)|=f(|t| ; z) .
$$


Therefore

$$
|f(t ; z)|= \begin{cases}f(|t| ; z), & t \geqslant 0 \\ f(|t| ;-z), & t<0 .\end{cases}
$$

The function $f(|t| ; z)$ can be expressed as

$$
f(|t| ; z)=-4 \pi \sum_{z_{j}>0} z_{j} n_{j}^{\infty} \exp \left(-z_{j}|t|\right)-4 \pi \sum_{z_{j}<0} z_{j} n_{j}^{\infty} \exp \left(-z_{j}|t|\right) .
$$

Since the first term decays to zero while the second term grows exponentially as $|t| \rightarrow \infty$,

$$
\frac{f(|t| ; z)}{|t|^{n}} \rightarrow \infty, \quad \text { as }|t| \rightarrow \infty .
$$

This limit remains unaltered if $z$ is replaced with $-z$. The desired result then follows from Equation (6). In fact, the nonlinearity of the Poisson-Boltzmann equation obeys assumption (A5) for all $n \in \mathbb{R}^{+}$.

Notation. A generic point in $\mathbb{R}^{d}$ is represented by $x$. If $F(\Omega)$ is a space of functions with domain $\Omega$, then

$$
F_{\text {loc }}(\Omega)=\{u: \Omega \rightarrow \mathbb{R} \mid u \in F(\Phi) \text { for each } \Phi \subset \subset \Omega\}
$$

and

$$
F_{0}(\Omega)=\{u: \Omega \rightarrow \mathbb{R} \mid u \in F(\Omega) \text { and } \mathcal{T} u=0 \text { on } \partial \Omega\}
$$

where $\mathcal{T}$ is the trace operator. The $d$-dimensional Lebesgue measure is given the symbol $m(\cdot)$. The (real) Lebesgue space $L^{2}(\Omega)$ and the (real) Sobolev space $H^{1}(\Omega)=W^{1,2}(\Omega)$ are equipped with their usual norms,

$$
\begin{aligned}
\|f\|_{L^{2}(\Omega)} & =\left(\int_{\Omega} f^{2} d m\right)^{1 / 2} \\
\|f\|_{H^{1}(\Omega)} & =\left(\int_{\Omega} f^{2} d m+\int_{\Omega}|D f|^{2} d m\right)^{1 / 2},
\end{aligned}
$$

where the gradient is to be interpreted as a weak derivative. The dual space of a generic Banach space $X$ will be denoted by $X^{*}$. The value of the functional $f \in X^{*}$ at $u \in$ $X$ is given the symbol $\langle f, u\rangle$. The symbols " $\rightarrow$ " and " $\rightarrow$ " represent strong and weak convergence, respectively.

\section{BACKGROUND THEORY}

Before commencing our search for the existence of a solution to Equations (4) and (5), we wish to discuss exactly what we mean by a solution. A classical $C^{2}(\Omega)$ solution is obviously desirable. However, what are the minimum requirements we can reasonably 
place on a solution to Equations (4) and (5)? After all, the fewer demands we place on a solution the easier it will be to prove its existence. Therefore we begin by defining a more general notion of a solution with the criteria that a classical solution must also be a generalised (weak) solution.

First assume $u$ is a classical solution of Equation (4). Then multiplying Equation (4) by $v \in C^{\infty}(\bar{\Omega})$ and integrating by parts gives

$$
\int_{\Omega} D u \cdot D v d m+\int_{\Omega} f(u) v d m=\int_{\Omega} g v d m+\int_{\partial \Omega} h v d S .
$$

If it is no longer assumed that $u$ is a classical solution of Equation (4), then does a function $u$ that satisfies Equation (7) exist in a broader class of functions? A function $u$ that satisfies Equation (7) for all smooth test functions $v$ shall be called a weak solution of Equations (4) and (5).

From Equation (7) it can be seen that we now only require $u \in C^{1}(\Omega)$. However, since a weak solution must only have integrable (in the sense of Lebesgue) first order partial derivatives, it need not possess a classical first derivative at all points in $\Omega$. That is, if the set of points in $\Omega$ where $D u$ does not exist has Lebesgue measure zero, then Equation (7) may still make sense. This enables us to interpret the derivatives in Equation (7) as weak derivatives. Then the minimum requirements on a weak solution are that it must have first order weak partial derivatives that are square integrable. Therefore we seek the existence of a weak solution $u \in H^{1}(\Omega)$ that solves Equation (7) for all smooth test functions $v$.

There are two main difficulties in establishing the existence of a solution in $H^{1}(\Omega)$ to Equation (7): Firstly, the rapid nonlinearity of $f$ means that it is not immediately apparent that Equation (7) makes any sense for $u, v \in H^{1}(\Omega)$. Secondly, the classical existence theorems of nonlinear functional analysis require that we work with coercive operators, and it is not obvious that the left hand side of Equation (7) induces a coercive operator in $H^{1}(\Omega)$. To overcome these difficulties, the following strategy will be employed:

1. define a related coercive operator by introducing a small perturbation to Equation (7);

2. truncate the nonlinear function $f$ to obtain a bounded operator;

3. recast this new perturbed and truncated equation in terms of an operator equation;

4. establish the existence of a solution to this operator equation;

5. take limits, in some sense, and prove that the limit function satisfies Equation 7.

Since the nonlinear function $f$ is assumed to be monotone, nonlinear monotone operator theory will be employed to establish the existence of a solution to Equation 7 . 
Definition 1: Let $A: X \rightarrow X^{*}$ be an operator on the real reflexive Banach space $X$. Then $A$ is pseudomonotone if and only if $u_{j} \rightarrow u$ in $X$ and

$$
\underset{j}{\limsup }\left\langle A u_{j}, u_{j}-u\right\rangle \leqslant 0
$$

implies

$$
\langle A u, u-v\rangle \leqslant \liminf _{j}\left\langle A u_{j}, u_{j}-v\right\rangle,
$$

for all $v \in X$.

LEMMA 1. Let $A: X \rightarrow X^{*}$ be a coercive operator on the real Banach space $X$, that is,

$$
\lim _{\|u\|_{X \rightarrow \infty}} \frac{\langle A u, u\rangle}{\|u\|_{X}}=+\infty
$$

Then for each $b \in X^{*}$, if there exists a solution to

$$
A u=b, \quad u \in X,
$$

the solution set is bounded.

Proof: See Zeidler [24].

The following classical theorem will be central in our proof of the existence of a weak solution to Equations (4) and (5).

THEOREM 1. (Brézis, 1968) Let $A: X \rightarrow X^{*}$ be a pseudomonotone, bounded and coercive operator on the real, separable and reflexive Banach space $X$. Then for each $b \in X^{*}$,

$$
A u=b, \quad u \in X,
$$

has a solution.

Proof: See Zeidler [24].

\section{Preliminary Results}

The solution strategy described in Section 2 is commenced by seeking a solution to the following perturbed (or, regularised) problem:

$$
\varepsilon \int_{\Omega} u v d m+\int_{\Omega} D u \cdot D v d m+\int_{\Omega} f(u) v d m=\langle b, v\rangle,
$$

for any fixed bounded linear functional $b$ and $\varepsilon>0$. Then for $V=H^{1}(\Omega)$ the bilinear form

$$
\ell_{\varepsilon}(u, v)=\varepsilon \int_{\Omega} u v d m+\int_{\Omega} D u \cdot D v d m,
$$

is well defined for $u, v \in V$ and

$$
v \mapsto \ell_{\varepsilon}(u, v)
$$


is a bounded linear functional on $V$. Thus it induces a map $L_{\varepsilon}: V \rightarrow V^{*}$ by the rule

$$
\left\langle L_{\varepsilon} u, v\right\rangle=\ell_{\varepsilon}(u, v) .
$$

The map $L_{\varepsilon}$ has a number of very useful properties, in particular it is coercive.

Lemma 2. Let $L_{\varepsilon}: V \rightarrow V^{*}$ be defined by Equation (9), then:

(i) $L_{\varepsilon}$ is a bounded linear operator with $\left\|L_{\varepsilon}\right\| \leqslant 2 M_{\varepsilon}$, where $M_{\varepsilon}=\max \{1, \varepsilon\}$;

(ii) $L_{\epsilon}$ is a coercive operator, that is,

$$
\lim _{\|u\|_{V} \rightarrow \infty} \frac{\left\langle L_{\varepsilon} u, u\right\rangle}{\|u\|_{V}}=+\infty
$$

for $\varepsilon>0$;

(iii) $L_{\varepsilon}$ is a monotone operator, that is,

$$
\left\langle L_{\varepsilon} u-L_{\varepsilon} v, u-v\right\rangle \geqslant 0,
$$

for all $u, v \in V$;

(iv) $L_{\varepsilon}$ is a symmetric operator, that is,

$$
\left\langle L_{\varepsilon} u, v\right\rangle=\left\langle L_{\varepsilon} v, u\right\rangle,
$$

for all $u, v \in V$;

(v) $L_{\varepsilon}$ is strongly continuous, that is, $u_{j} \rightarrow u$ in $V$ implies that $L_{\varepsilon} u_{j} \rightarrow L_{\varepsilon} u$ in $V^{*}$.

Proof: The proof of this lemma is straightforward, so only the key properties will be established. In what follows, $u, v \in V$.

(ii) The coercivity of $L_{\varepsilon}$ follows from

$$
\begin{aligned}
\left\langle L_{\varepsilon} u, u\right\rangle & =\varepsilon\|u\|_{L^{2}(\Omega)}^{2}+\|D u\|_{L^{2}(\Omega)}^{2} \\
& \geqslant m_{\varepsilon}\left(\|u\|_{L^{2}(\Omega)}^{2}+\|D u\|_{L^{2}(\Omega)}^{2}\right) \\
& =m_{\varepsilon}\|u\|_{V}^{2},
\end{aligned}
$$

where $m_{\varepsilon}=\min \{1, \varepsilon\}$. Hence

$$
\frac{\left\langle L_{\varepsilon} u, u\right\rangle}{\|u\|_{V}} \geqslant m_{\varepsilon}\|u\|_{V}
$$

and so $L_{\epsilon}$ is coercive for $\varepsilon>0$.

(v) Let $u_{j} \rightarrow u$ in $V$, then

$$
\begin{aligned}
\left\|L_{\varepsilon} u-L_{\varepsilon} u_{j}\right\|_{V^{*}} & =\left\|L_{\varepsilon}\left(u-u_{j}\right)\right\|_{V \cdot} . \\
& =\sup _{\|v\|_{v} \leqslant 1}\left\langle L_{\varepsilon}\left(u-u_{j}\right), v\right\rangle \\
& =\sup _{\|v\|_{v} \leqslant 1}\left\langle L_{\varepsilon} v, u-u_{j}\right\rangle .
\end{aligned}
$$


Therefore $\lim _{j \rightarrow \infty}\left\|L_{\varepsilon} u-L_{\varepsilon} u_{j}\right\|_{V^{*}}=0$, since $L_{\varepsilon} v \in V^{*}$. The linearity and symmetry of $L_{\varepsilon}$ were crucial here.

Following Webb [23], we observe that the term involving $f(u)$ does not induce a mapping from $V$ to $V^{*}$ because no growth restriction is made. To correct this we employ the truncation

$$
f_{n}(t)=\left\{\begin{array}{ll}
f(t), & \text { if }|f(t)| \leqslant n \\
n \frac{f(t)}{|f(t)|}, & \text { otherwise. }
\end{array} .\right.
$$

Then for fixed $n$

$$
\sigma_{n}(u, v)=\int_{\Omega} f_{n}(u) v d m
$$

is defined for all $u, v \in V$, and

$$
\begin{aligned}
\left|\sigma_{n}(u, v)\right| & \leqslant \int_{\Omega} n|v| d m \\
& \leqslant n\|v\|_{L^{2}(\Omega)}
\end{aligned}
$$

by Hölder's inequality. Thus

$$
v \mapsto \sigma_{n}(u, v),
$$

defines an element of $V^{*}$ and induces the map $S_{n}: V \rightarrow V^{*}$ by the rule

$$
\left\langle S_{n} u, v\right\rangle=\sigma_{n}(u, v) .
$$

Lemma 3. Let $S_{n}: V \rightarrow V^{*}$ be defined by Equation (10), then $S_{n}$ is a pseudomonotone operator.

PROOF: Let $u_{j} \rightarrow u$ in $V$ such that $\lim \sup _{j}\left\langle S_{n} u_{j}, u_{j}-u\right\rangle \leqslant 0$, for $n$ fixed. First observe that for all $v \in V$,

$$
\left\langle S_{n} u_{j}, u_{j}-v\right\rangle<\infty,
$$

since $\Omega$ is bounded. Therefore $\left\langle S_{n} u_{j}, u_{j}-v\right\rangle$ is a bounded sequence of real numbers. Consequently there exists a subsequence $\left\{u_{j_{k}}\right\} \subset\left\{u_{j}\right\}$ such that $[3]$

$$
\lim _{k}\left\langle S_{n} u_{j_{k}}, u_{j_{k}}-v\right\rangle=\liminf _{j}\left\langle S_{n} u_{j}, u_{j}-v\right\rangle
$$

Then for any subsequence $\left\{\bar{u}_{j_{k_{l}}}\right\} \subset\left\{u_{j_{k}}\right\}$ and all $v \in V$,

$$
\liminf _{l}\left\langle S_{n} \bar{u}_{j_{k_{l}}}, \bar{u}_{j_{k_{l}}}-v\right\rangle=\liminf \operatorname{in}_{j}\left\langle S_{n} u_{j}, u_{j}-v\right\rangle .
$$

The weak convergence $u_{j_{k}} \rightarrow u$ in $V$ implies there is another subsequence $\left\{u_{j_{k_{l}}}\right\}$ $\subset\left\{u_{j_{k}}\right\}$ such that $u_{j_{k_{l}}} \rightarrow u$ in $L^{2}(\Omega)$, by the Rellich-Kondrachov compactness theorem $[5,17]$. Moreover, we can assume that $u_{j_{k_{l}}} \rightarrow u$ pointwise almost everywhere in $\Omega$. The continuity of $f_{n}$ implies that $f_{n}\left(u_{j_{k_{l}}}\right) \rightarrow f_{n}(u)$ pointwise almost everywhere in $\Omega$. 
Furthermore, for any $v \in V,\left|f_{n}\left(u_{j_{k_{1}}}\right) v\right| \leqslant n|v|$. Since $n$ is fixed and $v \in L^{1}(\Omega), n|v|$ is integrable, therefore Lebesgue's Dominated Convergence Theorem gives

$$
\int_{\Omega} f_{n}(u) v=\lim _{l} \int_{\Omega} f_{n}\left(u_{j_{k_{l}}}\right) v .
$$

Assumptions (A2) and (A3) yield the sign condition $f(t) t \geqslant 0$ for all $t \in \mathbb{R}$, which also applies to $f_{n}$, so by Fatou's Lemma,

$$
\int_{\Omega} f_{n}(u) u \leqslant \liminf \int_{\Omega} f_{n}\left(u_{j_{k_{l}}}\right) u_{j_{k_{l}}} .
$$

Combining these,

$$
\begin{aligned}
\left\langle S_{n} u, u-v\right\rangle & =\int_{\Omega} f_{n}(u)(u-v) \\
& \leqslant \liminf \int_{\Omega} f_{n}\left(u_{j_{k_{l}}}\right)\left(u_{j_{k_{l}}}-v\right) \\
& =\liminf _{l}\left\langle S_{n} u_{j_{k_{l}}}, u_{j_{k_{l}}}-v\right\rangle,
\end{aligned}
$$

for all $v \in V$. Setting $\bar{u}_{j_{k_{l}}}=u_{j_{k_{l}}}$ in Equation (11) and using the above result, we obtain

$$
\left\langle S_{n} u, u-v\right\rangle \leqslant \liminf _{j}\left\langle S_{n} u_{j}, u_{j}-v\right\rangle,
$$

for all $v \in V$.

The following lemma is due to Webb [23].

Lemma 4. Under assumptions (A1) to (A3), if $\left\{u_{n}\right\}$ is a sequence in $V$ with $u_{n} \rightarrow u$ in $V$ and $\int_{\Omega} f_{n}\left(u_{n}\right) u_{n} \leqslant C$ for some $C>0$ and for all $n$, then $f(u) u \in L^{1}(\Omega)$ and $f_{n}\left(u_{n}\right) \rightarrow f(u)$ in $L^{1}(\Omega)$.

Proof: See Webb [23].

\section{THE EXISTENCE THEOREM}

THEOREM 2. Let $\Omega$ be a bounded domain of class $\mathcal{C}^{0,1}$ and let $f$ obey assumptions (A1) to (A4). Then for any $g \in L^{2}(\Omega)$ and $h \in L^{2}(\partial \Omega)$, there exists a unique $u \in H^{1}(\Omega)$ such that $f(u) \in L^{1}(\Omega)$ and $f(u) u \in L^{1}(\Omega)$ and

$$
\int_{\Omega} D u \cdot D v d m+\int_{\Omega} f(u) v d m=\int_{\Omega} g v d m+\int_{\partial \Omega} h \mathcal{T} v d S,
$$

for all $v \in H^{1}(\Omega) \cap L^{\infty}(\Omega)$ and for $v=u$.

Proof: Let $V=H^{1}(\Omega)$, then $V$ is a real Hilbert space and is therefore a separable and reflexive Banach space. Let $L_{\varepsilon}, S_{n}: V \rightarrow V^{*}$ be defined by Equations (9) and (10), respectively. It follows that $L_{\varepsilon}+S_{n}$ is pseudomonotone because the sum of a strongly 
continuous and a pseudomonotone operator is again pseudomonotone [24]. It can easily be shown that $L_{\varepsilon}+S_{n}$ is bounded for fixed $n$. We calculate

$$
\begin{aligned}
\left\langle\left(L_{\varepsilon}+S_{n}\right) u, u\right\rangle & =\left\langle L_{\varepsilon} u, u\right\rangle+\left\langle S_{n} u, u\right\rangle \\
& =\left\langle L_{\varepsilon} u, u\right\rangle+\int_{\Omega} f_{n}(u) u \\
& \geqslant\left\langle L_{\varepsilon} u, u\right\rangle
\end{aligned}
$$

by the sign condition $f_{n}(t) t \geqslant 0$ for all $t \in \mathbf{R}$, which follows from (A2) and (A3). Thus

$$
\lim _{\|u\|_{v \rightarrow \infty}} \frac{\left\langle\left(L_{\varepsilon}+S_{n}\right) u, u\right\rangle}{\|u\|_{v}}=+\infty,
$$

by the coercivity of $L_{\varepsilon}$, and so $L_{\varepsilon}+S_{n}$ is coercive for all $u \in V$ and $\varepsilon>0$. Hence for each $b \in V^{*}$ there exists a $u_{n}^{\varepsilon} \in V$ that satisfies

$$
\left(L_{\varepsilon}+S_{n}\right) u_{n}^{\varepsilon}=b
$$

by Theorem 1 .

By Lemma 1 there exists a $C_{1}^{\varepsilon}>0$ such that $\left\|u_{n}^{\varepsilon}\right\|_{v} \leqslant C_{1}^{\varepsilon}$. Therefore, as $V$ is a reflexive Banach space, there exists a subsequence $\left\{u_{n_{j}}^{\varepsilon}\right\} \subset\left\{u_{n}^{\varepsilon}\right\}$ such that $u_{n_{j}}^{\varepsilon} \rightarrow u^{\varepsilon}$ in $V$. Furthermore, $L_{\varepsilon}$ is bounded and thus $\left\|L_{\varepsilon} u_{n}^{\varepsilon}\right\|_{v^{*}} \leqslant C_{2}^{\varepsilon}$ for some $C_{2}^{\varepsilon}>0$. Equation (13) yields

$$
\int_{\Omega} f_{n}\left(u_{n}^{\varepsilon}\right) u_{n}^{\varepsilon} \leqslant\|b\|_{V \cdot} C_{1}^{\varepsilon}+C_{1}^{\varepsilon} C_{2}^{\varepsilon}
$$

Thus by Lemma $4, f\left(u^{\varepsilon}\right) u^{\varepsilon} \in L^{1}(\Omega)$ and $f_{n_{j}}\left(u_{n_{j}}^{\varepsilon}\right) \rightarrow f\left(u^{\varepsilon}\right)$ in $L^{1}(\Omega)$. The strong continuity of $L_{\varepsilon}$ implies $L_{\varepsilon} u_{n_{j}}^{\varepsilon} \rightarrow L_{\varepsilon} u^{\varepsilon}$ in $V^{*}$. It follows that for fixed $\varepsilon>0$ and for any $v \in V \cap L^{\infty}(\Omega)$, after letting $j \rightarrow \infty$,

$$
\left\langle L_{\varepsilon} u^{\varepsilon}, v\right\rangle+\int_{\Omega} f\left(u^{\varepsilon}\right) v=\langle b, v\rangle
$$

Observe that the convergence of the integral follows from Hölder's inequality. Equation (14) also holds for $v=u^{\varepsilon}$, because $u^{\varepsilon} \in V$ and by Lemma $4, f\left(u^{\varepsilon}\right) u^{\varepsilon} \in L^{1}(\Omega)$.

This concludes our search for a solution to the perturbed problem, Equation (8). We are now required to take the limit $\varepsilon \rightarrow 0$.

We claim that $\left\|u^{\varepsilon}\right\|_{V} \leqslant R$, where $R>0$ is independent of $\varepsilon$. To prove this we begin by obtaining a lower bound for $\int_{\Omega} f\left(u^{\varepsilon}\right) u^{\varepsilon}$. Indeed, (A1) to (A4) imply there exist $C_{f}^{+}, C_{f}^{-}>0$ such that $f(t) \geqslant C_{f}^{+} t$ if $t \geqslant 0$ and $f(t) \leqslant C_{f}^{-} t$ if $t<0$. Therefore $f(t) t \geqslant C_{f} t^{2}$ for all $t \in \mathbb{R}$, where $C_{f}=\min \left\{C_{f}^{+}, C_{f}^{-}\right\}$, and

$$
\int_{\Omega} f\left(u^{\varepsilon}\right) u^{\varepsilon} \geqslant C_{f}\left\|u^{\varepsilon}\right\|_{L^{2}(\Omega)}^{2} .
$$


This lower bound in conjunction with Equation (14) gives

$$
\begin{aligned}
\left\langle b, u^{\varepsilon}\right\rangle & =\varepsilon\left\|u^{\varepsilon}\right\|_{L^{2}(\Omega)}^{2}+\left\|D u^{\varepsilon}\right\|_{L^{2}(\Omega)}^{2}+\int_{\Omega} f\left(u^{\varepsilon}\right) u^{\varepsilon} \\
& \geqslant\left\|D u^{\varepsilon}\right\|_{L^{2}(\Omega)}^{2}+\int_{\Omega} f\left(u^{\varepsilon}\right) u^{\varepsilon} \\
& \geqslant\left\|D u^{\varepsilon}\right\|_{L^{2}(\Omega)}^{2}+C_{f}\left\|u^{\varepsilon}\right\|_{L^{2}(\Omega)}^{2} \\
& \geqslant \widetilde{C}_{f}\left\|u^{\varepsilon}\right\|_{V}^{2},
\end{aligned}
$$

where $\tilde{C}_{f}=\min \left\{1, C_{f}\right\}$. Finally, this calculation implies that

$$
\left\|u^{\varepsilon}\right\|_{V}^{2} \leqslant \widetilde{C}_{f}^{-1}\|b\|_{V} \cdot\left\|u^{\varepsilon}\right\|_{V} .
$$

Hence $\left\|u^{\varepsilon}\right\|_{V} \leqslant R$, where $R=\|b\|_{V \cdot} / \tilde{C}_{f}>0$ and is independent of $\varepsilon$.

Let $\varepsilon=\varepsilon_{n}$ where $\left\{\varepsilon_{n}\right\}$ monotonically decreases to zero as $n \rightarrow \infty, u^{n}=u^{\varepsilon_{n}}$ and $L_{n}=L_{\varepsilon_{n}}$. The bound on $u^{n}$ in $V$ implies that there exists a subsequence $\left\{u^{n_{j}}\right\} \subset\left\{u^{n}\right\}$ such that $u^{n_{j}} \rightarrow u$ in $V$ as $j \rightarrow \infty$. Furthermore, for all $v \in V \cap L^{\infty}(\Omega)$,

$$
\int_{\Omega} \varepsilon_{n} u^{n} v \rightarrow 0, \quad \text { as } n \rightarrow \infty,
$$

by Hölder's inequality. This is also true if $v=u^{n}$. Using the bound on $u^{n}$ in $V$, the bound on $L_{\varepsilon}$ obtained in Lemma 2 (which is independent of $\varepsilon$ for $0 \leqslant \varepsilon \leqslant 1$ ) and Equation (14) we obtain $\int_{\Omega} f\left(u^{n_{j}}\right) u^{n_{j}} \leqslant C$. So by Lemma 4 we have $f(u) u \in L^{1}(\Omega)$ and $f\left(u^{n_{j}}\right) \rightarrow f(u)$ in $L^{1}(\Omega)$, and as before for any $v \in V \cap L^{\infty}(\Omega)$ and for $v=u$,

$$
\int_{\Omega} f\left(u^{n_{j}}\right) v \rightarrow \int_{\Omega} f(u) v, \quad \text { as } j \rightarrow \infty
$$

Define the bounded linear map $L: V \rightarrow V^{*}$ via,

$$
\langle L v, w\rangle=\int_{\Omega} D v \cdot D w
$$

for $v, w \in V$. Then $u^{n_{j}} \rightarrow u$ in $V$ and Equation (15) imply that

$$
\left\langle L_{n_{j}} u^{n_{j}}, v\right\rangle=\int_{\Omega} \varepsilon_{n_{j}} u^{n_{j}} v+\left\langle L v, u^{n_{j}}\right\rangle \rightarrow \int_{\Omega} D u \cdot D v, \quad \text { as } j \rightarrow \infty,
$$

since $L v \in V^{*}$; this also holds for $v=u$. Finally, defining $\varepsilon=\varepsilon_{n_{j}}$ in Equation (14), using Equations (15) to (17) and sending $j \rightarrow \infty$ yields, for each $b \in V^{*}$,

$$
\int_{\Omega} D u \cdot D v+\int_{\Omega} f(u) v=\langle b, v\rangle
$$

for all $v \in V \cap L^{\infty}(\Omega)$ and for $v=u$. 
To prove that the solution to Equation (18) is unique, let $u_{1}$ and $u_{2}$ satisfy Equation (18) and observe that the strict monotonicity of $f$ implies

$$
\int_{\Omega}\left|D\left(u_{1}-u_{2}\right)\right|^{2}+\int_{\Omega}\left(f\left(u_{1}\right)-f\left(u_{2}\right)\right)\left(u_{1}-u_{2}\right)>0,
$$

for $u_{1} \neq u_{2}$. Since $u_{1}$ and $u_{2}$ both satisfy Equation (18), they must satisfy

$$
\int_{\Omega} D\left(u_{1}-u_{2}\right) \cdot D v+\int_{\Omega}\left(f\left(u_{1}\right)-f\left(u_{2}\right)\right) v=0,
$$

for all $v \in V \cap L^{\infty}(\Omega)$. Setting $v=u_{1}-u_{2}$ we obtain a contradiction unless $u_{1}=u_{2}$.

It remains to show that the functional defined by

$$
\langle b, v\rangle=\int_{\Omega} g v d m+\int_{\partial \Omega} h \mathcal{T} v d S,
$$

is an element of $V^{*}$ for $g \in L^{2}(\Omega)$ and $h \in L^{2}(\partial \Omega)$. To this end, for $v \in V$ with $\|v\|_{V} \leqslant 1$,

$$
\langle b, v\rangle \leqslant\|g\|_{L^{2}(\Omega)}+C\|w\|_{V},
$$

by Hölder's inequality and the Trace Theorem $[1,17]$, where $w \in V$ with $\mathcal{T} w=h$. Therefore $b \in V^{*}$. Finally, Casas and Fernández in [4, Lemma 2] and Kufner in [17, Theorem 6.9.2], give that $\mathcal{T} v \in L^{2}(\partial \Omega) \cap L^{\infty}(\partial \Omega)$ whenever $v \in V \cap L^{\infty}(\Omega)$. It can now easily be shown that $b \in\left(V \cap L^{\infty}(\Omega)\right)^{*}$.

\section{THE REgULARITY THEOREM}

In this section we show that the weak solution of Equation (4) exhibits higher regularity than a function in $H^{1}(\Omega)$. For simplicity the test functions shall be chosen in the space $H_{0}^{1}(\Omega) \cap L^{\infty}(\Omega)$. As a result, the regularity of the solution will be investigated only in the interior of $\Omega$, that is, away from $\partial \Omega$.

THEOREM 3. Let $\Omega$ be a bounded domain of class $\mathcal{C}^{0,1}$ and let $f$ obey assumptions (A1) to (A3) and (A5) with $g \in L^{2}(\Omega)$. Suppose furthermore that there exists $u \in H^{1}(\Omega)$ such that $f(u) \in L^{1}(\Omega)$ and $f(u) u \in L^{1}(\Omega)$ and

$$
\int_{\Omega} D u \cdot D v d m+\int_{\Omega} f(u) v d m=\int_{\Omega} g v d m
$$

for all $v \in H_{0}^{1}(\Omega) \cap L^{\infty}(\Omega)$ and for $v=u$. Then $u \in H_{\mathrm{loc}}^{2}(\Omega) \cap L^{\infty}(\Omega)$ and satisfies

$$
-\Delta u+f(u)=g,
$$

almost everywhere in $\Omega$. Furthermore, for each open subset $U \subset \subset \Omega$ we have the estimate

$$
\|u\|_{H^{2}(U)} \leqslant C\left(\|g\|_{L^{2}(\Omega)}+\|u\|_{L^{2}(\Omega)}+\|f(u)\|_{L^{2}(\Omega)}\right),
$$

the constant $C$ depending only on $U$ and $\Omega$. 
PROOF: Assumption (A5) implies that for each $n$ there exists $R_{n}>0$ such that

$$
|f(t)| \geqslant|t|^{n}, \quad \text { for } t \in \mathbb{R} \text { with }|t| \geqslant R_{n} .
$$

Introduce the set

$$
\Omega_{R_{n}}=\left\{x \in \Omega|| u(x) \mid \geqslant R_{n}\right\} .
$$

By assumption $u \in L^{1}(\Omega)$, now assume that $u \in L^{n}(\Omega)$ for some fixed $n \in \mathbb{N}$. We wish to show that $u \in L^{n+1}(\Omega)$. To this end,

$$
\begin{aligned}
\int_{\Omega}|u|^{n+1} & =\int_{\Omega_{R_{n}}}|u|^{n}|u|+\int_{\Omega \mid \Omega_{R_{n}}}|u|^{n}|u| \\
& \leqslant \int_{\Omega}|f(u) u|+R_{n}^{n} \int_{\Omega}|u| \\
& <\infty,
\end{aligned}
$$

by Equation (22) and the hypotheses of the theorem. Therefore $u \in L^{n+1}(\Omega)$. It then follows from the principle of mathematical induction that $u \in L^{n}(\Omega)$ for all $n \in \mathbb{N}$. Since $\Omega$ is bounded, we also have $u \in L^{\infty}(\Omega)$.

The monotonicity of $f$ plus the boundedness of $\Omega$ also ensure $f(u) \in L^{\infty}(\Omega)$. The proof of the theorem now follows directly from standard linear elliptic regularity theory $[5$, $8,20]$.

\section{Discussion}

Having proven Theorem 2, we are left pondering the question: in what sense does our weak solution satisfy Equations (4) and (5)? In particular, defining a normal derivative operator in $H^{1}(\Omega)$ is extremely problematic, so in what sense does our weak solution satisfy the Neumann boundary condition? These questions have been answered by Casas and Fernández [4] who prove that, if $g \in H^{1}(\Omega) \cap L^{\infty}(\Omega)$ and $h \in L^{2}(\partial \Omega) \cap L^{\infty}(\partial \Omega)$, then $u \in H^{1}(\Omega)$ satisfies Equation (12) if and only if $u$ satisfies Equations (4) and (5) in the sense of distributions. Note that Theorem 2 is sufficient to prove that a numerical solution to Equations (4) and (5) may be sought via the finite element method. This is significant because the boundary conditions are purely Neumann.

Equation (18) was obtained with no mention of boundary conditions. In particular our results are also valid for Dirichlet boundary conditions. In fact, the problem is dramatically simplified if a solution is sought in $H_{0}^{1}(\Omega)$ since it can be shown, via the Poincaré-Friedrichs inequality, that in this space the Laplacian induces a coercive operator. Hence there is no longer a need for regularisation, and the only assumptions on the nonlinearity that are required for existence are continuity and the sign condition $f(t) t \geqslant 0$ for all $t \in \mathbb{R}$. Also, a variational technique may be more appropriate when a solution is sought in $H_{0}^{1}(\Omega)$. 
Even greater regularity of the solution to Equation (4) can be obtained from Theorem 3. This follows from the imbedding of $W^{k, p}$ in $C$, the space of continuous functions. Therefore since $u \in H_{\mathrm{loc}}^{2}(\Omega)$, and if $d=1,2,3$, then $u \in C(\Omega)[1,17]$.

Finally, Theorems 2 and 3 also remain valid for uniformly elliptic second order equations of the form,

$$
\sum_{|\alpha|,|\beta| \leqslant 1}(-1)^{|\alpha|} D^{\alpha}\left(a_{\alpha \beta}(x) D^{\beta} u(x)\right)+f(x, u(x))=g(x),
$$

where $\alpha, \beta$ are $d$-dimensional multiindicies with $a_{\alpha \beta} \in C^{1}(\bar{\Omega})$ and $a_{\alpha \beta}=a_{\beta \alpha}$, and in addition to (A1)-(A5), $f$ is assumed to be measurable in its first argument [23].

\section{REFERENCES}

[1] R.A. Adams, Sobolev spaces (Academic Press, New York, 1975).

[2] J.G. Azorero, I.P. Alonso and J.P. Puel, 'Quasilinear problems with exponential growth in the reaction term', Nonlinear Anal. 22 (1994), 481-498.

[3] A. Browder, Mathematical analysis: An introduction (Springer-Verlag, New York, 1996).

[4] E. Casas and L.A. Fernández, 'A Green's formula for quasilinear elliptic operators', $J$. Math. Anal. Appl. 142 (1989), 62-73.

[5] L.C. Evans, Partial differential equations, Graduate Studies in Mathematics 19 (American Mathematical Society, Providence, R.I., 1998).

[6] D. De Figueiredo and J. Gossez, C. R. Acad. Sci. Paris Sér I 308 (1989), 277-280.

[7] H. Fujita, 'On the nonlinear equations $\Delta u+e^{u}=0$ and $\partial v / \partial t=\Delta v+e^{v '}$, Bull. Amer. Math. Soc. 75 (1969), 132-135.

[8] D. Gilbarg and N.S. Trudinger, Elliptic partial differential equations of second order, (2nd edition) (Springer-Verlag, Berlin, 1983).

[9] J.P. Gossez and P. Omari, 'A necessary and sufficient condition of nonresonance for a semilinear Neumann problem', Proc. Amer. Math. Soc. 114 (1992), 433-442.

[10] C.P. Gupta, 'Perturbations of second order linear elliptic problems by unbounded nonlinearities', Nonlinear Anal. 6 (1982), 919-933.

[11] C.P. Gupta and P. Hess, 'Existence theorems for nonlinear noncoercive operator equations and nonlinear elliptic boundary value problems', J. Differential Equations 22 (1976), 305-313.

[12] P. Hess, 'A strongly nonlinear elliptic boundary value problem', J. Math. Anal. Appl. 43 (1973), 241-249.

[13] M. Holst, Multilevel methods for the Poisson-Boltzmann equation, (Ph.D. Thesis) (Numerical Computing Group, University of Illinois at Urbana-Champaign, 1993).

[14] S. Hu and N.S. Papageorgiou, 'Nonlinear elliptic problems of Neumann-type', Period. Math. Hungar. 40 (2000), 13-29.

[15] R.J. Hunter, Foundations of colloid science (Oxford University Press, Oxford, 2001).

[16] J.W. Jerome, 'Consistency of semiconductor modeling: an existence/stability analysis for the stationary van Roosbroeck system', SIAM J. Appl. Math. 45 (1985), 565-590. 
[17] A. Kufner, O. John and S. Fučik, Function spaces (Noordhoff International Publishing, Leyden, 1977).

[18] J. Mawhin, J.R. Ward Jr. and M. Willem, 'Variational methods and semi-linear elliptic equations', Arch. Rational. Mech. Anal. 95 (1986), 269-277.

[19] F. Papalini, 'A quasilinear Neumann problem with discontinuous nonlinearity', Math. Nachr. 250 (2003), 82-97.

[20] M. Renardy and R.C. Rogers, An introduction to partial differential equations, Texts in Applied Mathematics 13 (Springer-Verlag, New York, 1993).

[21] I. Rubinstein, Electro-diffusion of ions, SIAM Studies in Applied Mathematics 11 (SIAM, Philadelphia, 1990).

[22] M. E. Taylor, Partial differential equations, Applied Mathematical Sciences 117 (Springer-Verlag, New York, 1996).

[23] J.R.L. Webb, 'Boundary value problems for strongly nonlinear elliptic equations', $J$. London Math. Soc (2) 21 (1980), 123-132.

[24] E. Zeidler, Nonlinear functional analysis and its applications $I I / B$ (Springer-Verlag, New York, 1990).

Particulate Fluids Processing Centre

Department of Mathematics and Statistics

The University of Melbourne

Victoria 3010 , Australia

e-mail: jrlooker@ms.unimelb.edu.au 